\title{
Leibniz y la noción de sustancia corpórea en el período medio (1677-1695)
}

\author{
Rodolfo Fazio $^{1}$
}

Recibido: 1 de octubre de 2015 / Aceptado: 2 de julio de 2016

Resumen. En el presente trabajo analizamos la reforma que Leibniz propone entre 1677 y 1695 en la noción de cuerpo y, a partir de ello, esclarecemos el concepto de sustancia corpórea que presenta en esos años. En primer lugar, desarrollamos las críticas que esgrime contra la concepción geométrica del cuerpo propia de los filósofos modernos; en segundo lugar, examinamos los cambios que propone en dicha noción y su caracterización en clave de fuerza primitiva pasiva; en tercer lugar, estudiamos la definición hilemórfica de la sustancia corpórea que ofrece y evaluamos el lugar que ella ocupa en su esquema metafísico.

Palabras claves: cuerpo; Leibniz; metafísica; sustancia corpórea.

\section{[en] Leibniz's account of corporeal substance in the middle years (1677-1695)}

\begin{abstract}
In the present work we analyze Leibniz's reform of the notion of body between 1677 and 1695 and, from there, we clarify the concept of corporeal substance. First, we develop his critiques to the modern conception of body; second, we examine the changes he proposes to that notion and his redefinition in terms of primitive passive force; third we study his hylemorphic characterization of corporeal substance and we evaluate its place in his metaphysics.
\end{abstract}

Keywords: body; Leibniz; metaphysics; corporeal substance.

Sumario. 1. Introducción; 2. Leibniz contra los modernos: la crítica a la concepción geométrica del cuerpo; 3. El cuerpo reformado: la reducción a la fuerza primitiva pasiva; 4. La noción de sustancia corpórea y la ofensiva contra el cartesianismo; 5 . Referencias bibliográficas.

Cómo citar. Fazio, R. (2017): “Leibniz y la noción de sustancia corpórea en el período medio (16771695)", en Revista Anales del Seminario de Historia de la Filosofia 34 (1), 105-125. 


\section{Introducción}

Yo no elimino el cuerpo, sino que lo restituyo a aquello que es. Leibniz a De Volder (GP II, 275)

La noción de sustancia corpórea constituye uno de los mayores enigmas de la metafísica de Leibniz. Este concepto, de gran importancia en su proyecto filosófico, sufre variaciones significativas a lo largo de su pensamiento que han sido evaluadas por los exégetas de modos muy diversos. En líneas generales, las lecturas tradicionales han propuesto una transición desde una posición juvenil, donde se acepta que la naturaleza está conformada por sustancias corpóreas, hasta los escritos maduros, en los cuales se niega la sustancialidad de los cuerpos. Entre los intérpretes contemporáneos es Glenn Hartz (2008) quien formuló con precisión el mayor interrogante para las lecturas clásicas: ¿por qué, entonces, la sustancia corpórea sigue apareciendo incluso hasta los últimos escritos de Leibniz? En esta pregunta se recogen los ecos de las críticas esgrimidas por autores que, frente a las primeras exégesis de la metafísica leibniziana, proponen interpretaciones alternativas que buscan dar cuenta de la razón por la que las sustancias corpóreas persisten en el sistema maduro, tales como Smith (2003), Phemister (2005), Arthur y Loptson (2006), Rutherford (2008), Garber (2009) o el mismo Hartz (2007).

La polémica acerca del estatus de la sustancia corpórea en la metafísica de Leibniz recorre todos los períodos de su pensamiento, pero encuentra un primer punto de inflexión en el llamado período medio (1677-1695)². En efecto, la lectura idealista tradicional, según la cual los cuerpos se reducen a percepciones y apetitos de sustancias simples, no pareciera representar adecuadamente la posición leibniziana de esos años, puesto que, tal como ha puesto de relieve Garber (1985), el filósofo alemán adopta una concepción de la sustancia corpórea en términos hilemórficos, a saber, como forma sustancial y materia prima o, en clave leibniziana, como fuerza primitiva activa y fuerza primitiva pasiva. El objetivo de nuestra investigación radica en comprender la reforma que Leibniz propone entre 1677 y 1695 respecto de la noción de cuerpo y, a partir de allí, esclarecer el concepto de sustancia corpórea de esos años. Con tal fin dividiremos el trabajo en tres momentos. En primer lugar, analizaremos las críticas que Leibniz esgrime contra las concepciones modernas del cuerpo. En segundo lugar, estudiaremos su definición reformada del mismo. En tercer lugar, examinaremos cómo entiende la sustancia corpórea y cuál es el lugar que ella ocupa en su metafísica del período medio a la luz de sus críticas al cartesianismo.

\section{Leibniz contra los modernos: la crítica a la concepción geométrica del cuerpo}

Entre 1677 y 1695 Leibniz lleva adelante una revisión del concepto moderno de cuerpo. En particular, cuestiona una tesis común a los pensadores del siglo XVII, a saber, que la extensión, esto es, la cualidad geométrica de extenderse en ancho, largo

Fijamos el período medio desde el regreso de la estancia de Leibniz en Paris en 1677 hasta las primeras publicaciones sobre temas de metafísica de 1694 y 1695, a saber De primae philosophiae emendatione et de notione substantiae (1694), el Système nouveau de la nature et de la communication des substances (1695) y el Specimen dynamicum (1695). 
y profundidad, constituya un atributo primitivo de los cuerpos o, en otros términos, que ellos sean esencialmente extensos y, a la vez, cosas existentes. Si bien la crítica se cristaliza como un ataque a la res extensa cartesiana, en verdad se trata, como veremos, de una ofensiva que Leibniz dirige tanto contra Descartes como contra sí mismo ${ }^{3}$.

A fin de comprender la crítica de Leibniz es imprescindible explicitar los elementos que operan detrás de sus argumentos: por una parte, la noción de cuerpo que él mismo defendiera en su juventud (1663-1672) y, por otra, dos tesis que establece en el período parisino (1672-1676) y utiliza en sus primeras pruebas, a saber, el carácter indeterminado de las cantidades continuas y la relatividad general del movimiento. Debido la complejidad de estos temas, en ambos casos nos limitaremos a exponer las tesis sin ingresar en los argumentos que se ofrecen en su defensa ${ }^{4}$.

Entre 1663 y 1671 Leibniz define al cuerpo como materia que existe en el espacio y, a partir de esta caracterización, establece las dos cualidades que reconoce como esenciales en esos años: la extensión y la impenetrabilidad -o antitipia ${ }^{5}$. La diferencia principal respecto de la res extensa cartesiana radica en que el filósofo alemán juzga necesario distinguir entre el espacio, por el cual el cuerpo es extenso, y la materia que lo ocupa, por la cual el cuerpo es impenetrable ${ }^{6}$. Ahora bien, hay dos particularidades de la posición del joven Leibniz que ayudan a comprender las críticas que desarrolla del período medio. Por una parte, el espacio se concibe como algo que se define por la sola extensión, esto es, como "el ente primero extenso o cuerpo matemático, que evidentemente no contiene más que tres dimensiones y es el lugar universal de todas las cosas" (A II, 1, 34). Cabe señalar que en el marco de su sistema juvenil Leibniz acepta que el espacio es sustancia y, como corolario de ello, que la magnitud, dirección y sentido de los movimientos de los cuerpos que cambian de lugar en él pueden determinarse de modo absoluto 7 . Por otra parte, dado

3 Aun cuando Leibniz presente su reforma de la metafísica como una superación del cartesianismo, ella se desarrolla con independencia de la del filósofo francés. En relación con la noción de cuerpo, el cambio que propone Leibniz no opera tanto sobre la noción cartesiana como sobre su propia concepción juvenil, cuyas fuentes no se encuentran en Descartes, sino en otros modernos tales como Hobbes o Gassendi; cf. Moll (1978; 1982; 1996).

4 Los exégetas modernos se han interesado principalmente por determinar la compatibilidad de las tesis defendidas entre 1677 y 1695 con el sistema maduro. Su vínculo con el período juvenil, empero, no ha recibido la misma atención. Aun cuando en las últimas décadas intérpretes como Mercer (2001), Beeley (1996), Garber (1982; 2009), Bassler (1999; 2002) han trabajado especialmente la metafísica leibniziana de juventud, la relación que ella tiene con período medio constituye todavía un interrogante abierto. En nuestra investigación proponemos comprender los cambios que se introducen en el período medio a la luz la posición leibniziana de juventud.

5 Leibniz sostiene que "el cuerpo es eso que está en el espacio, esto es, la cosa co-extensa al espacio" (A VI, 2, 167); cf. A VI, 1, 493 y A VI, 2, 305. El elemento distintivo de los cuerpos recibe el nombre de materia; tal como declara a Thomasius, "la materia es el ente que existe en el espacio o el ente coextenso al espacio" (A II, 1,34). Ahora bien, a partir de esta definición Leibniz concluye las dos cualidades esenciales que reconoce para los cuerpos durante su juventud: "Por lo tanto, es en estas dos cualidades que tanto los sabios como los profanos ubican la naturaleza del cuerpo: la extensión y la antitipia tomadas en conjunto [...]. Lo extenso no es más que ser en el espacio; la antitipia es no poder estar con otro cuerpo en el mismo espacio, sino que unos deben mover o detener a los otros. A partir de esto es patente que la naturaleza del cuerpo consiste en la extensión y la antitipia y, dado que nada hay en las cosas sin causa, no debe suponerse nada en los cuerpos cuya causa no pueda explicarse a partir de estos primeros constitutivos" (A II, 1, 36).

6 Cabe subrayar que impenetrabilidad del cuerpo, esto es, la propiedad de ocupar un espacio e impedir que otro cuerpo lo ocupe al mismo tiempo, no implica resistencia alguna al cambio locativo: el cuerpo por sí mismo no ofrece ningún impedimento o freno adquisición de movimiento en el choque con otro cuerpo.

$7 \quad$ En el período juvenil Leibniz defiende incluso que "el espacio es más sustancial que el cuerpo mismo; porque quitado el cuerpo, el espacio y su medida subsisten, lo que se llama vacío mientras ningún cuerpo no venga 
que la extensión es una cantidad continua y los cuerpos son esencialmente extensos -por existir en el espacio-, éstos también tienen tal característica; en otras palabras, el cuerpo se piensa como un cosa compuesta de partes homogéneas que comparten sus límites y, por lo tanto, es divisible al infinito en la medida en que todas sus partes son también extensas y, por ello, contienen recursivamente otras partes de la misma naturaleza. Es interesante notar que el reconocimiento de la extensión como un predicado primitivo de los cuerpos traslada el problema de la composición de las cantidades continuas al núcleo de su metafísica. Es por ello que el joven filósofo se ve obligado ya desde sus primeros años a ofrecer una solución al enigma. Hacia 1671 Leibniz formula su primera respuesta -la cual será abandonada en el período de Paris $^{8}$. En función de nuestra investigación, nos interesa sólo una de las tesis que asume, a saber, la aceptación de un infinito actual de partes en toda cantidad continua ${ }^{9}$. Esta tesis es acorde a su comprensión del cuerpo como una cosa extensa, pues las partes del cuerpo han de ser actuales, esto es, estar determinadas, por el hecho de tratarse de algo real y, asimismo, tales partes han de ser infinitas a fin de dar cuenta de la divisibilidad al infinito de las cantidades continuas.

Durante la estancia en Paris entre 1672 y 1676 Leibniz arriba a dos conclusiones que jugarán un papel protagónico en la reforma del concepto de cuerpo. En primer lugar, abandona la concepción absolutista del movimiento y se compromete con la relatividad general del mismo. En efecto, desde estos años en adelante Leibniz defiende que en todas las clases de cambio locativo, tanto inerciales como no inerciales, sólo puede determinarse de modo absoluto la variación de distancia entre dos cuerpos, pero nunca cuál de ellos está en movimiento y cuál está en reposo. Esto último depende del sistema de referencia que se elija, lo cual se reduce a una cuestión pragmática: ha de preferirse siempre la hipótesis que haga mover menos cuerpos para explicar el mismo cambio de distancia ${ }^{10}$. En segundo lugar, Leibniz rechaza el infinito actual en las cantidades, tanto discretas como continuas, y defiende que en ellas el número de partes no es ni finito ni infinito, sino que se encuentra indeterminado, pues suponer que hay infinitas partes en una cantidad y que, además, están determinadas y son anteriores al todo que conforman es inconsistente, pues conduce a la violación del axioma de Euclides, el cual es concebido en la filosofía leibniziana como un principio reducible al principio de identidad y, por ello, algo que no puede negarse bajo ningún concepto ${ }^{11}$. Tanto el compromiso con la equivalencia

a suceder al primero; por el contrario, quitado el espacio, ningún cuerpo subsiste"(A II, 1, 11). Respecto del movimiento absoluto, el propio Leibniz reconoce en sus escritos posteriores que es una consecuencia ineludible de su filosofía de juventud. Como veremos en nuestro trabajo, este corolario es utilizado en los escritos postparisinos para criticar la noción moderna de cuerpo; cf. A VI, 4, 1968.

8 Sobre la solución juvenil de Leibniz al problema del continuo, cf. Beeley (1996), Bassler (1998b) y Arthur (2001). Sobre los cambios del período parisino, cf. Fazio (2016a).

$9 \quad$ El compromiso de Leibniz con el infinito actual de partes en las cantidades continuas es explicitado en los Fundamenta praedemostrabilia de la Theoria motus abstracti. Cf. A VI, 2, 264.

10 El primer ensayo donde Leibniz desarrolla su defensa de la relatividad de toda clase de movimiento es los Principia mechcanica (1675); cf. A VI, 3, 101-111. En el período medio puede verse reiterada en el Specimen dynamicum (1695), cf. GM VI, 247. Sobre este tema, cf. Fazio (2017).

11 El caso particular que lleva a Leibniz a rechazar el infinito actual en las cantidades es el estudio de las paradojas de los números infinito desarrolladas por Galileo en Discorsi e dimostrazioni matematiche intorno a due nuove scienze (1638). Apartándose de la interpretación galileana, Leibniz concluye la inconsistencia de las cantidades infinitas ya en sus primeros escritos parisinos, como la Accessio ad arithmeticam infinitorum (1672) y De minimo et maximo (1673). Cf. A III, 1, 15; A VI, 3, 98. Sobre el pasaje del concepto de infinito al de indeterminado en Leibniz, cf. Bassler (1998a). 
general de las hipótesis respecto del movimiento como el carácter indeterminado de las cantidades continuas son tesis que Leibniz establece en Paris y persisten hasta sus últimos escritos; asimismo, ellas constituyen las primeras herramientas en su crítica a la noción moderna de cuerpo.

El impacto que las tesis parisinas tienen en la noción leibniziana de cuerpo se evidencia ya en los primeros escritos del período medio. En efecto, a partir de 1677 Leibniz se distancia de su posición juvenil y cuestiona que la extensión sea esencial a los cuerpos. Con ello no niega ni que haya cuerpos ni que pueda predicarse de ellos la extensión, sino únicamente que la cualidad geométrica de extenderse en largo, ancho y profundidad se trate de un predicado primitivo. Durante el período medio Leibniz desarrolla dos líneas argumentativas para demostrar su tesis: la primera se funda en el carácter indeterminado y relativo de la extensión y del movimiento; la segunda, en la falta de unidad que afecta a las concepciones geométricas de los cuerpos. Si bien la segunda crítica es la más conocida, pues constituye la ofensiva principal que expone desde 1686 en adelante, la primera es más básica, ya que no sólo contiene las razones originales que lo conducen a revisar el concepto de cuerpo, sino que además es imprescindible para que la segunda sea concluyente, en particular, para evitar posibles salidas alternativas a los reparos leibnizianos, tales como afirmar unidades extensas indivisibles -Cordemoy- o una única cosa extensa-Spinoza-.

En el primer grupo de críticas Leibniz utiliza los resultados de sus estudios parisinos para cuestionar la concepción geométrica de los cuerpos. En Mira de natura substantiae corporeae (1683), resume su posición general sobre el tema del siguiente modo:

Aunque la extensión y el movimiento se comprenden más distintamente que otras cualidades, ya que las restantes deben explicarse a partir de ellas, ha reconocerse que ni la extensión ni el movimiento son comprendidas por nosotros de modo completamente distinto, ya sea porque estemos envueltos siempre en las dificultades de la composición del continuo y del infinito, ya sea porque no hay ninguna figura determinada en la naturaleza de las cosas ni un solo movimiento determinado. Y tal como sucede con el color y el sonido, así también la extensión y el movimiento son fenómenos y no atributos verdaderos de las cosas que contengan alguna naturaleza absoluta sin relación con nosotros (A VI, 4, 1465).

En líneas generales, Leibniz sostiene que la extensión no es una propiedad de las cosas mismas, sino que, al igual que sucede con las cualidades secundarias, se trata de un predicado que sólo puede definirse en relación con nosotros. Entre los distintos argumentos que presenta para fundamentar su tesis, puede diferenciarse uno de tipo indirecto, que se basa en sus estudios acerca del movimiento, y otro de tipo directo, que se funda únicamente en su propuesta de salida del laberinto del continuo. Veámoslos por separado.

Leibniz presenta un primer argumento en el ensayo Spatium et motus revera relationes (1677):

Si el espacio fuera una cosa que consista en la sola extensión y la naturaleza de la materia fuera llenar el espacio y el movimiento cambio en el espacio, entonces el movimiento sería ciertamente absoluto y respecto de dos cuerpos que se aproximan recíprocamente podría decirse cuál está en movimiento y cuál en reposo o, si ambos se mueven, a qué 
velocidad lo hacen. Y de allí se siguen las conclusiones que alguna vez mostré en la Teoría del movimiento considerado abstractamente. Pero, en verdad, el espacio no es una cosa, ni el movimiento algo absoluto, sino que consisten en una relación (A VI, 4, 1968).

Las tres premisas del argumento constituyen los elementos fundamentales que conforman la ontología juvenil de Leibniz: el espacio es algo extenso, el cuerpo es materia que existe en el espacio y el movimiento es cambio locativo. Ahora bien, Leibniz afirma que, aceptados estos presupuestos, se podría distinguir entre el cambio de los cuerpos respecto de otros cuerpos y el cambio respecto del espacio mismo, esto es, se seguiría el movimiento absoluto, lo cual contradice la relatividad general del movimiento ${ }^{12}$. A fin de evitar la contradicción, se concluye que el espacio no es una cosa, sino sólo una relación entre cosas. Con ello no se niega que su esencia sea la extensión, sino únicamente que se trate de algo existente -esto es, el espacio es extensión, pero es algo ideal-. Cabe señalar que se trata de una prueba indirecta porque no se pone en cuestión que el cuerpo sea algo extenso, sino aquello sobre lo que se fundamenta esta tesis, a saber, que el espacio mismo lo sea. Asimismo, la crítica afecta exclusivamente a la definición leibniziana de cuerpo, según la cual éste es algo extenso por existir en el espacio, pero no atenta contra la tradición cartesiana, la cual no tendría dificultades para evitar la objeción ${ }^{13}$.

Leibniz desarrolla una segunda prueba a partir de sus modificaciones en la comprensión de las cantidades continuas. En Corpus est modus tantum entis (167879), escrito cuya datación e interpretación es un tema de debate entre los intérpretes contemporáneos ${ }^{14}$, se presenta el siguiente argumento:

El cuerpo no es una sustancia, sino sólo un modo del ente o una apariencia coherente. Por cuerpo no entiendo lo que los escolásticos componen a partir de materia y cierta forma inteligible, sino lo que los seguidores de Demócrito llaman masa extensa [moles]. Digo que esto no es una sustancia, pues demuestro que si se considera la masa extensa como una sustancia, caeríamos en una contradicción que resulta del laberinto del continuo. Por ello debemos considerar: primero, que no puede haber átomos, ya que entran en conflicto con la sabiduría divina y, segundo, que los cuerpos mismos están realmente divididos en infinitas partes y, sin embargo, no en puntos. En consecuencia, [las partes] no pueden de ningún modo asignarse a un cuerpo, sino que cualquier porción de materia es un ente por accidente o, en otras palabras, está en flujo perpetuo. Pero si sólo decimos que los cuerpos son apariencias coherentes se pone fin a toda investigación acerca de lo infinitamente pequeño que no puede ser percibido (A VI, 4, 1637).

12 Cabe subrayar que Leibniz defiende que el movimiento es algo indeterminado en sí mismo, esto es, que nadie podría, ni siquiera una mente infinita, determinar en una variación de distancia entre cuerpos qué cuerpo está en movimiento y qué cuerpo está en reposo de modo absoluto (cf. A VI, 3, 111). Es por ello que la mera posibilidad del movimiento absoluto, permitida por la distinción entre espacio y cuerpo, alcanza para afirmar la contradicción con el principio de relatividad.

13 Este argumento no sirve como crítica a la res extensa de Descartes. En el sistema cartesiano el espacio es algo extenso y, al mismo tiempo, se afirma el carácter relativo del movimiento. Sin embargo, para que se concluya la posibilidad de movimiento absoluto es imprescindible un supuesto ausente en el cartesianismo, a saber, la distinción entre cuerpo y espacio.

14 El escrito no cuenta con marca externa alguna que permita su datación precisa; las razones esgrimidas en pos de una u otra fecha se basan en el contenido del escrito. La edición de la Academia propone fecharlo durante el viaje de Leibniz a Italia (1689-90). Arthur, por su parte, lo ubica en el período inmediatamente posterior a Paris (1678-79); cf. Leibniz, G. W., The Labyrinth of the continuum: writings on the continuum problem, op. cit., p. 416. Creemos, con Arthur, que el contenido del mismo revela la cercanía con las reflexiones parisinas. 
El argumento es altamente críptico -e incomprensible si no se lo lee en conjunto con los textos leibnizianos dedicados al tema del continuo--. Autores como Adams $(1994,236)$ han juzgado que el escrito muestra un compromiso con el fenomenalismo. El título, de hecho, parecería ir en esa línea. Sin embargo, una lectura atenta de la prueba revela que el objetivo de Leibniz es otro. Antes de analizar el argumento, es imprescindible considerar algunas cuestiones generales. Por una parte, Leibniz precisa al comienzo cuál es la noción de cuerpo que va a evaluarse. No es ni el cuerpo escolástico ni tampoco el cartesiano, sino aquel que se define como masa extensa (moles) $)^{15}$. Por otra parte, Leibniz introduce dos supuestos sobre los que no ofrece mayores explicaciones en el escrito -las razones para tales tesis hay que buscarlas en su período juvenil y parisino-: en primer lugar, advierte que el cuerpo así definido no puede ser atómico, sino que es divisible al infinito y, en segundo lugar, afirma que además es necesario suponerlo constituido de infinitas partes actuales, pero no puntos, pues no hay indivisibles en los cuerpos ${ }^{16}$. Con la definición del cuerpo como moles y esta doble precisión se evidencia que la noción que se evalúa críticamente en Corpus est modus tantum entis es nuevamente el concepto de cuerpo como materia que existe en el espacio y está infinitamente divida en acto. De este modo, el objetivo principal del opúsculo es mostrar que tal concepción del cuerpo no es capaz de escapar a las contradicciones del laberinto del continuo.

Teniendo en cuenta estas precisiones, el núcleo de la prueba podría reconstruirse del siguiente modo: (1) se supone que el cuerpo es masa extensa, (2) se sigue que tiene una cantidad de partes infinitas en acto -esto es, determinadas-; (3) sin embargo, no hay modo alguno de asignar una cantidad infinita determinada -tesis parisina-; (4) se produce la siguiente contradicción: el cuerpo como masa extensa ha de tener una cantidad infinita de partes determinadas, pero es imposible determinarlas; (5) se concluye que el cuerpo no es masa extensa. El argumento presupone las tesis parisinas de Leibniz, en particular, el carácter indeterminado de la extensión, esto es, que las partes que la componen no pueden determinarse de modo absoluto, sino que para ello es necesario establecer una relación con otra cosa que oficie de parámetro. Dado este inconveniente, Leibniz introduce lo que juzga es la solución al problema del continuo: reducir la masa extensa a algo ideal, es decir, algo que no existe por fuera de la mente que lo piensa. Ahora bien, con ello Leibniz no niega que haya cuerpos en general ni se compromete con un idealismo fenomenalista, puesto que todo el argumento opera bajo una concepción peculiar de cuerpo: la que Leibniz defendiera en su juventud. Tal cuerpo es, en suma, el que no es apto de existir como sustancia.

15 En la primera versión del texto Leibniz había escrito extensum y, posteriormente, lo corrige por el término técnico moles. Tal como indica Arthur (cf. Leibniz, G. W., The Labyrinth of the continuum: writings on the continuum problem, op. cit., p. 455), esta palabra es propia de la física de la época y se la utiliza para referirse a la cantidad de materia. Siguiendo la propuesta de traducción de Arthur y, asimismo, guiándonos por el término usado por el propio Leibniz en la primera versión escrito (extensum), creemos que con moles se refiere a la definición de cuerpo como una materia en el espacio, esto es, como una masa extensa. El hecho de que tal es la noción en debate se evidencia en el desarrollo del argumento.

16 Leibniz acepta en su juventud que es necesario introducir indivisibles en las cantidades continuas, pero defiende que ellos no son partes, sino sólo límites de tales cantidades. Con vistas a dar cuenta cómo esto es posible, en su Theoria motus abstracti presenta una comprensión híbrida del indivisible, según la cual ellos no son mínimos, sino que tienen una cantidad, esto es, tienen partes, pero no tienen partes separables o partes extra partes, razón por la cual sirven para establecer límites en las cantidades sin que sean parte de ellas; cf. A VI, 2, 264. Al igual que el infinito actual, los indivisibles serán abandonados en el período parisino; cf. A VI, 3, 97-98. 
El segundo grupo de críticas dirigidas a demostrar la imposibilidad de una res extensa se fundan en las consideraciones de Leibniz en torno a la unidad de la sustancia. A diferencia de las objeciones anteriores, el contrincante en este caso es Descartes. La preocupación leibniziana por la unidad sustancial o per se de los cuerpos, así como el fracaso de la tradición cartesiana en garantizarla, cobra fuerza a partir de la lectura de Cordemoy en 1685. Se la desarrolla en sus principales escritos metafísicos y en sus intercambios epistolares más importantes de esta época, tal como la correspondencia con Arnauld. Sin embargo, el núcleo de la crítica leibniziana se encuentra ya unos años antes de su lectura del atomista cartesiano. En An corpora sint mera phaenomena (1982-83) Leibniz expone el núcleo de su argumento:

(1) Supongo que lo que no tiene una unidad mayor que leños reunidos o apilados uno sobre otros no es propiamente un ente, sino entidades, aunque un nombre pueda suponerse para todos ellos (2) Supongo que no se comprende del cuerpo nada excepto la extensión, esto es, que tiene partes extra partes. (3) Finalmente, supongo que cada cuerpo está actualmente dividido en distintas partes, que también son cuerpos. De aquí se sigue: Primero, que no hay algo así como un cuerpo. Segundo, que no puede darse un cuerpo que no sea más que cuerpo y cuerpo. De aquí se sigue o bien que los cuerpos son meros fenómenos y no entidades reales o bien que hay algo distinto a la extensión en los cuerpos. Este argumento es exitoso incluso si la tercera suposición no se concede, siempre que se acepte que el solo contacto no hace una entidad (A VI, 4, 1464).

En primer lugar, Leibniz distingue entre la unidad de un ente (unidad sustancial o por sí) y la unidad de un conjunto de entes (unidad por agregación o accidental). En segundo lugar, supone que el cuerpo es res extensa y que la extensión constituye el único atributo suyo, esto es, restringe su crítica al cartesianismo. La conclusión que Leibniz extrae de estas premisas es presentada en la forma de una disyunción. Por un lado, afirma que, si no se reconoce en los cuerpos más que extensión, entonces de su divisibilidad al infinito se deriva que los cuerpos no son ni un ente, sino sólo agregados de ellos. Por tal razón, Leibniz afirma inmediatamente que no se trata de una sustancia, sino de un fenómeno, puesto que la pila de leños, a pesar de referirnos a ella con un nombre, no es una unidad sustancial, sino sólo una unidad por agregación, pues por fuera del pensamiento sólo hay leños. Por otra parte, plantea que, si se quiere garantizar la unidad de los cuerpos, habría que reconocer en ellos algo distinto a la extensión. Ahora bien, el interés de Leibniz es mostrar que, sea cual sea el disyunto que se prefiera, la conclusión es la misma: no es posible que exista algo como la res extensa cartesiana, dado que, si la naturaleza del cuerpo se reduce a la sola extensión, entonces no es una res por carecer de la unidad requerida, y si se quiere garantizar su sustancialidad, entonces es necesario reconocer que no puede ser sólo extensa.

El objetivo que Leibniz persigue con estas pruebas es fundamentalmente negativo, a saber, demostrar que la definición que la filosofía moderna ha ofrecido de los cuerpos es inadecuada, tanto en la versión que él mismo aceptara en su juventud como en la cartesiana. En efecto, el filósofo alemán juzga que el problema general radica en introducir la extensión como propiedad esencial del cuerpo. Ya sea que constituya su único atributo o no, ello conduce a dificultades insuperables: o bien viola el principio de relatividad general, o bien conduce a las contradicciones del laberinto del continuo, o bien no permite garantizar la unidad sustancial. Leibniz arriba de este modo a una 
de sus tesis más célebres, la cual es presentada ya a Arnauld en estos términos: "no existen más que las sustancias indivisibles con sus diferentes estados absolutamente reales" (A II, 2, 250). Ahora bien, cabe subrayar las conclusiones extraídas de los argumentos aquí presentados no implican que Leibniz se comprometa en estos años ni con que el cuerpo sea fenómeno ni tampoco con que la sustancia corpórea sea un agregado de sustancias. Precisar qué se entiende por cuerpo en el período medio posibilitará comprenderlo. Como veremos a continuación, entre 1677 y 1695 Leibniz ofrece una definición restringida de cuerpo como fuerza primitiva pasiva, cualidad que, a diferencia de la extensión, sí constituirá un "estado absolutamente real" de la sustancia indivisible y, asimismo, permitirá defender no sólo que hay sustancias corpóreas, sino además que toda sustancia finita es de tal clase.

\section{El cuerpo reformado: la reducción a la fuerza primitiva pasiva}

Entre 1677 y 1695 Leibniz propone una reforma en el concepto de cuerpo con la cual se aleja tanto de la noción cartesiana como de la que él mismo defendiera en su juventud. En una carta a Alberti de 1691 ofrece la siguiente presentación general:

Si la esencia del cuerpo consistiera en la sola extensión, entonces ella debería alcanzar para dar razón de todas las afecciones del cuerpo. Pero eso no es así. Nosotros subrayamos en la materia una cualidad que algunos llaman inercia natural, por la cual el cuerpo resiste al movimiento, de manera que hace falta emplear fuerza para imprimirlo [...] y que un cuerpo más grande es mucho más difícil de mover que uno pequeño (A II, 2, 395).

El reconocimiento de la resistencia natural como nota esencial del cuerpo es algo que Leibniz ya admite en su juventud e inicios del período medio como una posible salida para dar respuesta a los problemas propios de la física; por ejemplo, en las Regulae motus systematicae praestari mechanice (1677) declara que "todo procedería más simplemente si asumiéramos la inercia de la materia, a saber, que un cuerpo mayor resiste más" (A VI, 4, 1960). Sin embargo, Leibniz rechaza que esta propiedad sea esencial a los cuerpos hasta 1678, año en el que, con el nacimiento de la dinámica, invierte su posición ${ }^{17}$. Ahora bien, en principio el reconocimiento de la inercia natural como una cualidad primitiva de los cuerpos busca enfatizar que en ellos hay una resistencia al cambio. Pero, ¿no se trata, entonces, de una mera reintroducción de la inclinatio ad quietem de los escolásticos? Para lograr una comprensión adecuada de la tesis leibniziana es necesario analizar más detenidamente su caracterización del cuerpo en clave de vis patiendi y evaluarla en el contexto de su filosofía.

17 A pesar de que la datación del origen la dinámica leibniziana es un tema en debate entre los intérpretes, cf. Robinet $(1986,220-221)$ y Duchesneau $(1994,122)$, en función de nuestro tema alcanza con indicar que ya hacia 1680 Leibniz cuenta con los elementos que retomará en filosofía primera. Por una parte, en De corporum concursu (1678) el filósofo alemán establece por primer vez su argumento a favor distinción entre cantidad de movimiento y fuerza motriz. Por otra parte, en los escritos inmediatamente siguientes, tales como Principia mecánica ex metaphysicis dependere (1678-81) y Specimina de motus causa et de corporum qualitatibus(1678-81), Leibniz se interesa por demostrar que la fuerza motriz, a diferencia del movimiento, es algo ínsito en los cuerpos que puede determinarse de modo absoluto, esto es, con independencia del sistema de referencia elegido. Con esta reforma en el campo de la física, Leibniz comienza a repensar su concepción de cuerpo del período juvenil. 
Retomando parcialmente la terminología propia de la filosofía natural, a lo largo del período medio Leibniz defiende que la esencia del cuerpo no es ni la extensión ni la impenetrabilidad, sino la fuerza primitiva pasiva. Esta noción es presentada en sociedad en el Specimen dynamicum (1695):

Si se ha interpretado correctamente, la fuerza primitiva de soportar o resistir constituye lo mismo que se denomina en la escolástica materia prima, gracias a la cual resulta que un cuerpo no es penetrado por otro cuerpo y oponga al mismo una resistencia, y, a su vez, esté dotado de una cierta inercia, por así decir, esto es, de una repugnancia al movimiento, y no soporte por ello ser impulsado por la fuerza del agente, a no ser que quebrante algo en ésta (GM VI, 236-237) ${ }^{18}$.

A fin de comprender el concepto de fuerza pasiva primitiva, en primer lugar y con ayuda de algunas consideraciones propias de la dinámica analizaremos la noción de fuerza pasiva en general y, en segundo lugar, evaluaremos en qué radica su carácter primitivo.

En líneas generales con la reducción de la esencia de los cuerpos a la vis patiendi Leibniz busca dar con una cualidad más fundamental que la impenetrabilidad y la extensión que, no obstante, sea también capaz de dar cuenta de ellas. En este sentido afirma a Pellison que, a fin de explicar adecuadamente las leyes del movimiento "no queda sino recurrir a un principio más elevado de acción y de resistencia, del cual la extensión y la impenetrabilidad emanan cuando Dios no lo impide por un orden superior (A II, 2, 487). En efecto, Leibniz juzga que tal fuerza permite explicar las dos cualidades que en su juventud reconociera como esenciales a los cuerpos, las cuales en el período medio se transforman en derivadas. Veamos entonces las razones de ello.

Con la introducción de la fuerza pasiva como única cualidad esencial del cuerpo Leibniz se aleja radicalmente de su posición juvenil en un tema central. En sus escritos previos a 1678 el filósofo alemán acepta como propiedad fundamental de la materia la impenetrabilidad, esto es, el ocupar un lugar en el espacio e impedir que otro lo haga, pero en esos años, no obstante, rechaza explícitamente que la materia misma presente impedimento al cambio locativo, pues sólo un movimiento puede frenar otro movimiento, siendo el cuerpo indiferente a ello. Desde la redacción de $D e$ corporum concursu (1678) en adelante Leibniz acepta que los cuerpos son capaces de resistir al cambio por el simple hecho de ser cuerpos. En el ámbito de la filosofía natural, la introducción de la fuerza pasiva tiene la función de explicar la pérdida de cantidad de movimiento que se produce, por ejemplo, en el caso del choque de cuerpos en un sistema de péndulos ${ }^{19}$. En esta línea Leibniz declara en Principia mecánica ex metaphysicis dependere (1678-1681) lo siguiente:

Pero cuando consideré de qué modo en el universo puede darse razón de esto, lo que en todas partes experimentamos, a saber, que la velocidad disminuye con el aumento de la masa, tal como vemos que el mismo barco que baja por el río avanza más despacio

18 En los años anteriores al Specimen dynamicum Leibniz ya presenta esta definición a corresponsales tales como Bossuet y Pellison, donde nuevamente enfatiza su distinción con el concepto moderno de cuerpo; cf. A II, 2, 486-487 y 515-516.

19 Este es el caso particular que en De corporum concursu conduce a Leibniz a distinguir entre las nociones de fuerza motriz y cantidad de movimiento. Cf. DCC, 116-124 y Duchesneau (1994, 123-130). 
cuanto más cargado está, dudé ciertamente y, habiendo sido todos mis intentos en vano, descubrí que eso que se dice inercia de los cuerpos no puede deducirse de la noción de materia y movimiento asumida inicialmente, donde la materia se entiende como eso que se extiende o llena el espacio y el movimiento se entiende como cambio de espacio o lugar, sino que, además de lo que se deduce a partir de la sola extensión y de su variación y modificaciones, es necesario añadir y reconocer que en los cuerpos hay nociones o ciertas formas que se dicen inmateriales o independientes de la extensión, las que pueden llamarse potencias, por las cuales la velocidad se ajusta a la magnitud. Esta potencia no consiste ni en el movimiento, ni en el conato o inicio del movimiento, sino en la causa o razón intrínseca del movimiento (A VI, 4, 1980).

Ahora bien, Leibniz caracteriza la fuerza pasiva como aquello por lo que el cuerpo "no soporta ser impulsado por la fuerza de un agente, a no ser que quebrante algo en ésta" (GM VI, 237). De este modo, la vis patiendi se presenta como un obstáculo del cuerpo que, para ser superado, exige la impresión de una fuerza, la cual, asimismo, también se ve disminuida por ello. En este sentido la fuerza de resistencia es más que impenetrabilidad: se trata de una resistencia activa al cambio (concepto ausente en Descartes, Hobbes o Spinoza) ${ }^{20}$. Es por ello que Leibniz la explica, por ejemplo, en Definitiones cogitationesque metaphysica (1678) en estos términos: "resistente es eso que actúa en eso por lo que padece" (A VI, 4, 1394), y en el Specimen dynamicum sostiene que "la pasión de todo cuerpo es espontánea" (GM VI, 251). De esta forma, así como la fuerza activa consiste en la capacidad del cuerpo de pasar de un estado a otro, la pasiva no es más que la capacidad del cuerpo de impedir tal cambio. Pero ello no implica para Leibniz ni movimiento ni reposo. Tal como resume su comprensión de la inercia natural en De ipsa natura (1698), ella es sólo "una constancia natural opuesta al cambio" 21 . En consecuencia, Leibniz se aparta de la comprensión kepleriana de la inercia natural en la medida en que en el contexto de una posición relativista como la leibniziana la expresión "tendencia al reposo" no está bien definida, puesto que movimiento y quietud son atributos de los cuerpos que siempre han de establecerse en función de algún marco de referencia determinado y, por ello, siempre pueden intercambiarse con una alteración de este último.

Leibniz juzga que la fuerza pasiva es un estado del cuerpo que se erige como principio no sólo de resistencia, sino también de pluralidad ${ }^{22}$. En principio, tal como la define en De affectibus (1679): "pasión es el estado de la cosa por el que impide que algo se siga de su naturaleza [...]; potencia pasiva es la posibilidad de pasión" (A VI, 4, 1428). La vis patiendi se piensa, entonces, como un freno natural al cambio

20 La idea básica es que la resistencia exige un tipo de actividad y, por ello, no se puede deducir ni de la materia de los modernos (que es pasividad absoluta) ni del movimiento (que tampoco es una actividad, sino sólo cambio de posición relativa). Seguimos en este punto a Garber (2009, 117 y 136-137).

$21 \quad$ El propio Leibniz aclara la cuestión en De ipsa natura (1698): "la materia resiste al movimiento mediante una especie de inercia natural, como perfectamente la llamó Kepler, de modo que no es indiferente al movimiento y al reposo, según se suele pensar vulgarmente, sino que para ser movida requiere una fuerza activa proporcional a su tamaño [...]. Y muestra que de aquí se siguen las leyes del movimiento, las que son muy diferentes de las que se obtienen si se admite en el cuerpo y la materia sólo la extensión y la impenetrabilidad. Y así como en la materia la inercia natural se opone al movimiento, del mismo modo es inherente al cuerpo mismo o, mejor dicho, a toda sustancia una constancia natural opuesta al cambio (GP IV, 510-511, sub. prop.) Sobre la concepción de la inercia en Leibniz en comparación con Kepler y Newton, cf. Bernstein (1981).

22 En este punto seguimos a Garber (2009, 122-123). 
de estados de una cosa: se trata de su limitación intrínseca. En este sentido Leibniz juzga que la fuerza pasiva es una condición necesaria para que haya una pluralidad de cosas en el universo. Es por ello que, bajo la definición restringida de cuerpo como fuerza primitiva pasiva propia del período medio, se afirme, como veremos, que toda sustancia finita o creada es corpórea. Ahora bien, Leibniz juzga ya desde estos años -aunque sus explicaciones más acabadas se encuentren en el período maduro- que la pluralidad es un concepto más fundamental que el de extensión, pues esta última noción es subsidiaria de la primera, en tanto la cualidad de extenderse en largo, ancho y profundidad se explica a partir de una relación de continuidad que pueden surgir entre los diversos estados simultáneos de una pluralidad de cosas. Al igual que sucediera con la impenetrabilidad, el objetivo leibniziano es mostrar que la extensión no se trata de una cualidad que pertenezca a las cosas mismas: ella es una característica que refiere a la relación entre una multiplicidad de cosas, por lo que no constituye una propiedad primitiva de ningún sustrato, sino que es una idea que formamos a partir de la percepción de una particular relación entre estados de cosas que no son en sí mismas extensas.

La nota que distingue la noción de fuerza pasiva propia de la metafísica de aquella de la filosofía natural es el carácter primitivo. Sobre este tema nos limitaremos a indicar el núcleo de la posición leibniziana, sin ingresar en una justificación de la misma -que constituye sin duda el punto más áspero de su metafísica madura-. A lo largo del período medio Leibniz caracteriza la fuerza primitiva en oposición a la fuerza derivativa. En efecto, a diferencia de esta última, que se caracteriza como un estado momentáneo que únicamente refiere al estado próximo, ya sea causándolo en el caso de la fuerza activa o impidiéndolo en la pasiva, la fuerza primitiva se caracteriza por ser un único estado invariante que explica todas las acciones y pasiones de una misma cosa. En efecto, creemos que con la noción general de vis primitiva Leibniz no busca sino garantizar que el sustrato o sujeto que actúa o resiste en todos los cambios es uno y el mismo. De allí que el carácter primitivo de la potencia de resistir no sea algo que se derive de la dinámica leibniziana (ni se requiera en absoluto en filosofía natural), sino que responde a interrogantes propios de su metafísica, en particular, al problema de la unidad de la sustancia. En virtud de ello Leibniz sostiene que las fuerzas pasivas que se determinan, por ejemplo, en el choque de los cuerpos no son más que modificaciones de aquella capacidad primitiva de las cosas: tal es el sentido de la definición ofrecida en el Specimen dynamicum, donde se caracteriza la fuerza primitiva pasiva como aquello de lo que resultan las fuerzas secundarias estudiadas por la física.

En suma, la reforma del concepto de cuerpo consiste en reconocer que las dos cualidades que se aceptaban como constitutivas de su esencia, a saber, la impenetrabilidad y la extensión, no son más que propiedades derivadas que no pertenecen verdaderamente a tal noción. El único atributo fundamental, del cual Leibniz cree poder derivar los otros dos, es la capacidad primitiva o invariante de resistencia al cambio. Como ya hemos indicado, con ello no se elimina completamente tales propiedades: de hecho, el cuerpo que estudia el filósofo natural no sólo es algo resistente (fuerza derivativa), sino también extenso e impenetrable. Sin embargo, así como es peligroso en física apelar a las entelequias o a la materia prima a la hora de explicar los fenómenos observables de la naturaleza, Leibniz cree que es riesgoso en metafisica recurrir a las cualidades derivadas, tales como la extensión. Esta precisión es capital para el debate acerca de las sustancias corpóreas, porque la pregunta por el 
estatus ontológico de los cuerpos en el período medio pertenece exclusivamente a la metafísica y ha de evaluarse en función de aquello que Leibniz juzga que constituye la esencia de los cuerpos. En conclusión y retomando la pregunta inicial, ¿qué es un cuerpo para Leibniz durante el período medio? Las respuestas es precisa en estos años: aquello que tenga fuerza primitiva pasiva ${ }^{23}$.

\section{La noción de sustancia corpórea y la ofensiva contra el cartesianismo}

Entre 1677 y 1695 Leibniz defiende una teoría hilemórfica de la sustancia corpórea, según la cual ella se caracteriza por ser un sustrato o sujeto cuya esencia radica en tener fuerza primitiva tanto activa como pasiva. Antes de iniciar nuestro estudio de este concepto, es conveniente precisar algunas cuestiones respecto de la noción de sustancia en general que Leibniz presenta en el período medio. Ella es caracterizada a partir de dos notas. En primer lugar, se trata de un sujeto o sustrato que tiene la capacidad de pasar de un estado a otro cuando no hay impedimento para ello (esto es, aquello que tiene autosuficiencia causal de sus modificaciones). En segundo lugar, es un único y mismo sustrato capaz de causar todas sus distintas modificaciones, es decir, una potencia invariante que se conserva en cada uno de los sustratos y produce todos sus cambios. De este modo, la sustancia se define como una unidad invariante capaz de causar todos los distintos cambios de estado cuando no hay impedimento para ello o, en terminología leibniziana, fuerza activa primitiva. Tanto Dios como las sustancias finitas están incluidos, a juicio de Leibniz, en esta definición.

En el breve ensayo Mira de natura substantiae corporeae (1683) Leibniz se dedica exclusivamente al concepto de sustancia corpórea y ofrece una primera aproximación al mismo. En el pasaje central de la obra afirma lo siguiente:

En verdad, no puede decirse a qué sujeto pertenece el movimiento y, por lo tanto, nada en el movimiento es real más allá de la fuerza y potencia ínsita en las cosas, esto es, más allá de tener una constitución tal que de ella se siga el cambio de fenómenos según ciertas reglas determinadas. La extensión no pertenece a la sustancia del cuerpo como tampoco el movimiento, sino sólo la materia o principio de pasión o de la naturaleza limitada y la forma o principio de acción o de la naturaleza ilimitada. Pues toda creatura contiene tanto lo limitado como lo ilimitado. Lo limitado respecto del pensamiento distinto y del poder irresistible, y lo ilimitado respecto del pensamiento confuso y la acción difusa. Pues toda alma o, mejor dicho, toda sustancia corpórea es omnisciente confusamente y omnipotente difusamente. En efecto, nada sucede en el mundo que no se perciba confusamente y no hay esfuerzo que no se extienda hasta el infinito. No se dice impropiamente que la forma sea el acto primario. Cada cosa creada tiene materia y forma, esto es, es corpórea. Cada sustancia es inmortal. Cada sustancia corpórea tiene un alma. Cada alma es inmortal. Es probable que cada alma, mejor dicho, cada sustancia corpórea haya existido siempre desde el comienzo de las cosas. Una pila o entidad por agregación tal como un conjunto de piedras no debe llamarse una sustancia corpórea, sino sólo un fenómeno. La sustancia

23 En el período medio Leibniz se refiere a la fuerza primitiva pasiva como materia prima y a la derivativa como materia segunda. La primera se piensa como aquello que se completa con una forma sustancial y conforma la sustancia corpórea. La materia segunda, por su parte, se piensa como un agregado de sustancias. Sobre la comparación de la fuerza pasiva primitiva y derivativa con la materia primera y segunda de los escolásticos, cf. Adams (1994, 324). 
corpórea no tiene ninguna extensión definida. Hay tantas almas como átomos sustanciales o sustancias corpóreas (A VI, 4, 1465-1466, sub. prop) ${ }^{24}$.

Si se atiende a lo precisado en esta cita, puede advertirse, en primer lugar el rechazo de Leibniz de que la esencia de la sustancia corpórea consista en la extensión. Es decir, no se trata de una res extensa, sino de algo que ha de comprenderse en términos hilemórficos: es un compuesto de materia o principio de pasión y forma o principio de acción. En segundo lugar, Leibniz afirma que toda sustancia creada es una sustancia corpórea, es decir, que toda sustancia finita es un compuesto de forma y materia o, en clave cartesiana, de alma y cuerpo. En tercer lugar, Leibniz sostiene que la forma y la materia de la sustancia corpórea constituyen una verdadera unidad: ellas no son agregados ni tienen una unidad accidental, sino que conforman una genuina unidad atómica o sin partes.

Aun cuando Leibniz retoma en múltiples ocasiones el vocabulario específico del aristotelismo y del cartesianismo, los conceptos de forma y materia o de alma y cuerpo son comprendidos en su filosofía de un modo particular ${ }^{25}$. En efecto, la forma, alma o principio de acción es concebida como fuerza primitiva activa y la materia, cuerpo o principio de pasión, como fuerza primitiva pasiva. Tal es, por ejemplo, la caracterización ofrecida en De mundo praesenti (1686):

la sustancia corpórea tiene partes y especies. Las partes son la materia y la forma. La materia es el principio de la pasión, o sea, la fuerza primitiva de resistencia [...]. La forma sustancial es el principio de la acción, o sea, la fuerza primitiva de actuar (A VI, $4,1507-1508)$.

Un primer punto a considerar para comprender esta noción radica en la relación que las fuerzas pasivas guardan con las activas. En principio, Leibniz reconoce que es posible, y de hecho existente, una cosa que se defina sólo por su fuerza primitiva activa, a saber, la sustancia infinita o Dios. Sin embargo, rechaza la posibilidad de que exista algo cuya naturaleza consista en la sola fuerza primitiva pasiva. Por el contrario, ésta siempre reclama una fuerza activa que la complete. Ello se debe a que la fuerza pasiva se concibe como la posibilidad de impedir el paso de un estado a otro, lo cual no puede comprenderse ni existir de modo independiente a la tendencia a realizar tal cambio. De allí que Leibniz reitere una y otra vez que no hay cuerpo o fuerza de resistencia sin alma o fuerza de actuar. En este sentido afirma en $D e$ mundo praesenti que "es necesario que esta forma sustancial se encuentre en todas las sustancias corpóreas, las cuales son una unidad por sí” (A VI, 4, 1508, sub.

24 La caracterización hilemórfica de la sustancia corpórea ya se perfila en los textos inmediatamente posteriores a 1678. Por ejemplo, en Definitiones cotitationesque metaphysicae (1678-1680) afirma que "cuerpo es una sustancia que puede actuar o padecer. Materia es el principio de la pasión. Forma es el principio de la acción" (A VI, 4, 1399). Sobre el concepto general de sustancia en el período medio, cf. Fazio (2016b).

25 Leibniz alterna el vocabulario según el contexto. Utiliza las duplas fuerza activa y fuerza pasiva, mente y cuerpo o forma y materia. La elección de los términos varía fundamentalmente según el contexto de discusión. Lo importante en cada caso es no perder de vista la peculiar comprensión leibniziana de ellos. Por ejemplo, en su carta a Bernoulli de 1698 sostiene sobre el uso del vocabulario que "He preferido decir lo incompleto es lo activo sin lo pasivo y lo pasivo sin lo activo, más que decir que es la materia sin forma o viceversa, para referirme a la explicación misma más que a la cosa que ha de ser explicada, adelantándome de alguna manera a tu consejo, pues, en efecto, al común de los modernos le ofende menos el nombre actividades que el de formas" (GM III, 551). 
propio) y en el Specimen dynamicum que "por lo que respecta a la fuerza activa (que, con algunos, se podría llamar virtud), ésta es doble, a saber: en tanto que primitiva está presente en toda sustancia corpórea por sí [...]" (GM VI, 236, sub. propio). Tal como explica en el período maduro a Bernoulli a partir de la contraposición con la materia segunda:

La materia en sí misma o mole que se denomina materia prima no es una sustancia ni un agregado de sustancias, sino algo incompleto (GM III, 537).

Leibniz juzga que la materia prima sin forma sustancial es sólo una abstracción. No es ni sustancia ni tampoco fenómeno, sino algo incompleto que refiere únicamente a un estado de las sustancias finitas que no puede darse por sí solo, a saber, la resistencia al cambio.

Aceptado que hay una sustancia que sólo consiste en fuerza primitiva activa y rechazado que pueda haber materia sin forma, el siguiente punto a considerar es si puede haber forma sin materia en el caso de las sustancias creadas. En otras palabras, ¿podría darse una pluralidad de sustancias cuya esencia radique en la sola fuerza de actuar? Sobre este punto Leibniz es muy claro en el período medio: tal como declara en el Specimen dynamicum, "en toda sustancia existe la fuerza de actuar y, si ha sido creada, también la de padecer" (GM VI, 247). La razón de ello consiste en que la fuerza pasiva constituye la limitación de la sustancia finita por la cual no sólo se diferencia de la sustancia infinita, sino también de las otras sustancias finitas. La multiplicidad de sustancias exige, entonces, que el alma esté siempre unida a un cuerpo, esto es, que su acción tenga límites. Es en este sentido que en Mira de natura substantiae corporeae afirma que "cada cosa creada tiene materia y forma, esto es, es corpórea" (A VI, 4, 1466). De este modo, la fuerza pasiva no pertenece a la esencia de la sustancia en general, pero sí a la de la sustancia finita. Es importante advertir, entonces, que tanto la fuerza activa como pasiva son elementos constitutivos de las sustancias creadas: ninguna puede removerse, pues sin la primera no habría nada y sin la segunda no se trataría ya de algo finito, sino de Dios mismo.

Resumiendo lo considerado hasta aquí, Leibniz juzga que en las sustancias finitas no puede haber fuerza pasiva sin activa ni activa sin pasiva y es en razón de ello que durante el período medio se afirma que toda sustancia finita es corpórea. En conexión con este punto es importante subrayar que no se trata de un agregado de dos fuerzas, sino de una única fuerza primitiva ${ }^{26}$. En efecto, el mayor peligro en este caso es sustancializar lo que no son más que dos aspectos de una misma potencia primitiva. Tal como le indica el propio Leibniz a Pellison en 1692, al responder a la objeción de un cartesiano que permanece en el anonimato, la forma sustancial no es algo que se añade al cuerpo o materia prima y lo constituya en sustancia:

Hasta el momento no he encontrado nada que explique mejor la naturaleza de la sustancia material que la fuerza de actuar y de resistir [...]. [El objetor] dice que tengo otro pensamiento sobre los cuerpos, a saber, que hay formas sustanciales. Pero este pensamiento viene con el precedente. La forma sustancial no es otra cosa que la fuerza

26 Como lo presenta Phemister (2005, p. 40-46), son dos caras de la misma moneda que no pueden separarse, pues, tal como advertirá a Bernoulli en 1698, "incompleto es lo activo sin lo pasivo y lo pasivo sin lo activo" (GM III, 551). 
primitiva. La dificultad sobre la unidad de los cuerpos no debe descuidarse. Ella ha apremiado al señor Cordemoy y lo ha forzado a recurrir a los átomos (A II, 2, 598) ${ }^{27}$.

Leibniz advierte que no hay que pensar a la sustancia corpórea como un cuerpo al que se añade una forma sustancial, pues, en verdad, no hay dos cosas que se unan, sino un solo sustrato o sujeto cuya esencia consiste en ser una fuerza primitiva limitada de actuar. Ahora bien, este planteo sólo tiene sentido bajo la acepción restringida de cuerpo que opera en estos años, a saber, como fuerza primitiva pasiva.

Cabe recordar que Leibniz no acepta que existan las fuerzas primitivas, sino únicamente sujetos cuya esencia son tales fuerzas, esto es sustratos que tienden y resisten al cambio, que actúan y padecen. La distinción entre el sustrato o cosa y su esencia es capital para evitar la sustancialización de lo que para Leibniz no son más que conceptos que, aun cuando expresan las cualidades primitivas de los sustratos, no subsisten sin ellos. En una carta a Pellison establece esta diferencia central de modo claro:

Nadie podrá ofenderse si se toma la sustancia en abstracto por la fuerza primitiva, la cual permanece siempre la misma en el mismo cuerpo y hace nacer sucesivamente fuerzas accidentales y acciones particulares [...]. Sin embargo, es verdad que la sustancia en concreto es algo distinto de la fuerza, puesto que es el sujeto tomado con esa fuerza (A II, 2, 487).

La distinción entre acción y pasión es abstracta, así como también lo es la misma esencia de la sustancia. En la naturaleza sólo existen las cosas, sustratos o sujetos que actúan y padecen. Es por ello que la unidad de la fuerza primitiva se funda, en definitiva, en que se trata de una misma cosa que actúa y padece. La idea de que la sustancia corpórea conforma un uno por sí se mantendrá en el período maduro, pero para entonces se afirma en un contexto distinto y más dificultoso, pues se refiere a la unidad entre una mónada dominante y un cuerpo orgánico (un agregado de mónadas). Sobre esa cuestión, que constituye el punto más álgido del debate contemporáneo, no nos pronunciaremos en esta investigación.

La cuestión de la unidad entre la fuerza primitiva pasiva y activa es abordada en los escritos leibnizianos de fines del período medio en el marco del debate cartesiano acerca de la unión entre la mente y el cuerpo. Este problema no es tratado explícitamente en la presentación de su metafísica en el Système nouveau, obra en la que Leibniz sólo se pronuncia respecto de la comunicación entre las sustancias y presenta su teoría de la armonía preestablecida. Sin embargo, tanto en el Specimen dynamicum como, por ejemplo, en la Eclaircissement du nouveau système (1696) advierte que su sistema filosófico permite dar cuenta de uno de los mayores interrogantes legado por Descartes a la filosofía del siglo XVII: ¿qué soy? En la segunda parte del Specimen dynamicum resume la cuestión del siguiente modo:

La naturaleza no suficientemente conocida del cuerpo, y más aún la de la sustancia en general, había dado como resultado [...] que filósofos sin duda insignes de nuestro tiempo, al ubicar la noción de cuerpo sólo en la extensión, se viesen obligados a recurrir a Dios para explicar la unión entre alma y el cuerpo, y aún más la comunicación de los

27 Sobre las objeciones que le comunica Pellison, cf. A II, 2, 569-570. 
cuerpos entre sí. Pues se ha de confesar que es imposible que la mera extensión, reducida a nociones geométricas, sea capaz de acción y pasión; por consiguiente, les pareció que esto era suficiente: que al pensar el hombre en mover un brazo, Dios, como por un acuerdo primigenio, mueve el brazo por él y que, por el contrario, existiendo el movimiento en la sangre y en los espíritus, Dios produce la percepción en el alma. Pero estas mismas cosas, puesto que son ajenas a la forma correcta de filosofar, habrían debido advertir a sus autores que se apoyaban en un principio falso, y que no habían definido correctamente la noción de cuerpo, de la que se obtenían como consecuencias semejantes resultados. Así pues, demostraremos que en toda sustancia existe la fuerza de actuar y, si ha sido creada, también la de padecer; que la noción de extensión no es de por sí completa, sino una referencia a algo que se extiende, de lo cual es difusión o réplica continuada, y a tal punto se presupone una sustancia del cuerpo que encierra el poder de actuar y de resistir, y está presente en toda masa corpórea, y que la difusión de ésta está contenida en la extensión. Partiendo de aquí, también encenderemos una nueva luz para explicar la unión del ama y el cuerpo (GM VI, 246-247)

Leibniz juzga que si se aborda la pregunta bajo la concesión de que el cuerpo es una res extensa, el problema se torna irresoluble y conduce a salidas tales como el ocasionalismo de Malebranche. Es la mala comprensión de la unión alma-cuerpo lo que conduce a que la comunicación se torne inexplicable.

En un escrito previo a su reforma de la metafísica, titulado Distinctio mentis et corporis (1677-1678), Leibniz analiza el problema cartesiano y adelanta una posible salida, la cual constituirá el núcleo de su respuesta al interrogante en 1695:

Sé que soy y, sin embargo, dudo que haya algún cuerpo en la naturaleza de las cosas; por lo tanto, no soy cuerpo. Este argumento es débil, si no se muestra que la extensión constituye por sí una sustancia completa; en efecto, si se acepta eso, es manifiesto que a partir de aquella sustancia nunca puede nacer el pensamiento. Pero eso todavía no fue demostrado. Ciertamente sabemos que el pensamiento no es un modo de la extensión, pero desconocemos si acaso no es un modo de la sustancia, de la cual la misma extensión sea otro de sus modos (A VI, 4, 1368, sub. Prop.).

En líneas generales, Leibniz advierte que si la extensión constituyese la esencia de los cuerpos -es decir, el argumento de la cera-, entonces podría concluirse que, dado que de ella no se deriva el pensamiento, el cuerpo es distinto del alma. Sin embargo, Leibniz no parece conformarse con la prueba cartesiana y declara que hay que probar todavía que la extensión pueda constituir una sustancia. Ahora bien, en la última oración propone una solución alternativa al interrogante: que tanto la extensión como el pensamiento sean modos de una misma sustancia. Esta idea, que, más allá de la terminología, guarda cierto parecido con la propuesta spinozista -ya conocida por Leibniz en estos años-, constituye la base de la respuesta ensayada hacia 1695, donde los conceptos de sustancia y cuerpo se presentan redefinidos en términos de fuerza ${ }^{28}$.

28 El propio Leibniz ubica en sus estudios sobre dinámica el origen que permite dar respuesta al interrogante de la unión entre alma y cuerpo. En efecto, en la Eclaircissement du nouveau système declara: "Mis ensayos dinámicos tienen relación con esto [la comunicación entre el alma y el cuerpo], donde ha habido que profundizar en la noción de sustancia corpórea, que ubico en la fuerza de actuar y de resistir y no en la extensión" (GP IV, 499). 
¿En qué consiste el núcleo de la respuesta leibniziana al enigma cartesiano? Sentadas las bases de la ontología de Descartes, en la que se afirma una sustancia infinita (Dios) y dos clases de sustancias finitas, a saber, la sustancia pensante y la sustancia extensa, el desafío asumido por el cartesianismo consistió en explicar cómo se unen esos dos tipos de sustancia finitas para formar al hombre, esto es, una sustancia cuya esencia es tanto el pensamiento como la extensión. La solución que Leibniz propone al interrogante con su noción de sustancia corpórea es radical, pues consiste en reformar las bases ontológicas que lo generan. Como punto de partida, el filósofo alemán rechaza, como vimos, que sea posible algo como una res extensa o, en otros términos, que la extensión pueda constituir un atributo esencial de una sustancia. Con esto, empero, no busca reducir la extensión a un modo de la sustancia pensante. Por el contrario, el núcleo de su propuesta consiste en afirmar que, así como no hay res extensa, tampoco hay res cogitans, sino que lo que Descartes distinguiera como dos clases de sustancias finitas se subsumen en una única clase, compuesta siempre de cuerpo y mente. Esta tesis se comprende únicamente si se acepta que el cuerpo no es extensión, sino fuerza pasiva primitiva, y la mente no es pensamiento, sino fuerza activa primitiva ${ }^{29}$. En suma, el resultado de la reforma leibniziana de la metafísica arroja un sistema donde se reconoce una sustancia infinita (Dios) y una solo tipo de sustancia finita, la cual se compone de cuerpo y alma y es denominada en el período medio sustancia corpórea, esto es, compuesto de fuerza primitiva pasiva y activa. Bajo este esquema ontológico el problema de la unión no se responde, sino que se anula. Ahora bien, un grave problema que afecta a la propuesta de Leibniz -y se revela en los debates que emprende para defender su posición metafísica- radica en el uso de términos propios del cartesianismo para referirse a nociones que le son completamente ajenas. En efecto, la fuerza primitiva pasiva y activa, a pesar de recibir el nombre de "cuerpo" y "mente", no tienen ni origen ni traducción a la metafísica cartesiana. En este punto la solución de Leibniz se diferencia de otras tales como la de Spinoza o la de Malebranche, pues no se construye desde Descartes, sino sólo contra Descartes.

A fin de marcar la distancia existente entre su sistema y el cartesiano, Leibniz presenta en 1695 un término en función del cual va a comenzar a reordenar su metafísica: la monada. En una carta a L'Hospital de julio de 1695 y en el marco de una discusión respecto del Système nouveau recién publicado, introduce este nombre para referirse a las unidades básicas de la naturaleza:

la clave para mi posición sobre este tema consiste en la consideración de eso que es genuinamente una unidad real, una mónada (A III, 6, 451).

Creemos que con esto Leibniz intenta superar las interferencias lingüísticas e indicar que su noción de sustancia finita no es ni la sustancia corpórea cartesiana ni tampoco la sustancia pensante, sino otra cosa. La estrecha relación de este concepto

29 Leibniz no quiere que se identifique su concepción de la fuerza activa primitiva o entelequia con el pensamiento de la filosofía cartesiana. En este sentido, por ejemplo, le advierte a Arnauld que no hay que limitar la noción: "creo haber mostrado que toda sustancia es indivisible y que, por consiguiente, toda sustancia corpórea debe tener un alma o al menos una entelequia que tenga analogía con el alma, pues, de otra manera, lo cuerpos no serían más que fenómenos. Asegurar que toda sustancia que no es divisible (es decir, según mi opinión, toda sustancia en general) es un espíritu y debe pensar, me parece incomparablemente más osado y falto de fundamento que la conservación de las formas" (A II, 2, 252). 
con la metafísica del período medio se revela en el hecho de que las primeras definiciones que ofrece de la mónada no es sino la misma que diera en el período medio de la sustancia corpórea, a saber, un compuesto de fuerza primitiva activa y pasiva $^{30}$. Con la introducción de un nuevo vocabulario el concepto de sustancia corpórea sufrirá un desplazamiento en sus escritos maduros. En efecto, en los escritos posteriores a 1695 ya no se define en términos hilemórficos, sino que se caracteriza por ser una mónada asociada a un cuerpo orgánico; siguiendo a Phemister (2001), en el período maduro puede hablarse entonces de una concepción hilozoica de la sustancia corpórea ${ }^{31}$. A pesar de este corrimiento semántico, el debate acerca de la sustancia corpórea del período medio constituye un punto ineludible a la hora de comprender la metafísica madura de Leibniz, la cual se construye a partir de las nociones establecidas entre 1677 y 1695.

\section{Referencias bibliográficas}

\section{Fuentes}

Leibniz, G. W., Sämtliche Schriften und Briefe, Hrsg. von der Berlin-Brandenburgischen Akademie der Wissenschaften und der Wissenschaften in Göttingen, Darmstadt-LeipzigBerlin, Akademie Verlag, 1923-sig [citado como A].

Leibniz, G. W., Die philosophischen Schriften, ed. C. I. Gerhardt, 7 vols., Berlín, Wiedeman Buchhandlung, 1875-1890 [citado como GP].

Leibniz, G. W., Mathematische Schriften, 7 vols, ed. C. I Gerhardt, Berlin, A. Asher, 18481863. [citado como GM].

Leibniz, G. W., La réforme de la dynamique, ed. Michel Fichant, París, Vrin, 1994 [citado como DCC]

Leibniz, G. W., The Labyrinth of the continuum: writings on the continuum problem, trad. R. Arthur, New Haven/London Yale University Press, 2001.

Leibniz, G. W., Obras filosóficas y cientificas II: Metafísica, A. L. González (ed.), Granada, Editorial Comares, 2010.

Leibniz, G. W., Obras filosóficas y cientificas VII: Escritos cientificos, Juan Arana (ed.),

30 En 1703 Leibniz define a la mónada en estos términos: "Hablando con propiedad y rigor, tal vez no deberíamos decir que la entelequia primitiva actúa sobre la masa de su cuerpo, sino que se junta con la potencia pasiva primitiva a la que completa y con la que constituye la mónada" (GP II, 250). La misma caracterización es reiterada a Des Bosses en 1706. "La materia prima o to dynamikon prôton pathêtikon, prôton hypokeimenon, es decir, la potencia primitiva pasiva o principio de resistencia no consiste en la extensión sino en la exigencia de extensión y completa a la entelequia o potencia activa primitiva para que aparezca la sustancia acabada o mónada, en la que están virtualmente contenidas las modificaciones" (GP II, 306).

31 Phemister $(2001,80-81)$ plantea que el cambio del hilomorfismo por el hilozoísmo se encuentra ya en 1683 . En favor de esta tesis pueden hallarse referencias durante el período medio en las que Leibniz parece ya vincular la noción de cuerpo no con la fuerza pasiva primitiva sino con la de cuerpo orgánico (un agregado de sustancias). En efecto, algunos pasajes de la correspondencia con Arnauld como también, por ejemplo, la discusión con Fardella sobre la sustancia corpórea (A VI, 4, 1670-1671) abonan esta tesis. Sin embargo, son pocas las ocasiones en las que Leibniz se compromete en estos años con esta definición de cuerpo. En efecto, hasta 1695 se sigue identificando al cuerpo con la fuerza primitiva pasiva y la concepción predominante es la noción hilemórfica de la sustancia corpórea. Por ejemplo, en la correspondencia con Pellison, Bossuet o Alberti, y en escritos tales como De mundo praesenti o el mismo Specimen dynamicum -todos posteriores a 1683-, Leibniz ubica la esencia del cuerpo en la sola fuerza primitiva pasiva. Creemos que la ambigüedad se debe a la falta de precisión terminológica y que, por ello, es conveniente limitar la discusión respecto de la noción hilezoica de la sustancia corpórea para los textos maduros, donde se precisan conceptos claves, como los de cuerpo orgánico y mónada dominante, ausentes en el período medio, sin las cuales no puede abordarse adecuadamente la cuestión. 
Granada, Editorial Comares, 2009.

Leibniz, G. W., Obras filosóficas y cientificas XIV: Correspondencia I, J. A. Nicolás y M.R. Cubells (ed.), Granada, Editorial Comares, 2010

Leibniz, G. W., Escritos filosóficos, ed. Ezequiel de Olaso, Buenos Aires, Editorial Charcas, 1982.

\section{Bibliografía secundaria}

Adams, R. (1994), Leibniz: Determinist, Theist, Idealist, Oxford, Oxford University Press.

Arthur, R. y Loptson, P. (2006), "Leibniz's Body Realism: Two Interpretations", Leibniz Review, ${ }^{\circ} 16$, pp. 1-42.

Bassler, O. (1998a), "Leibniz on the Indefinite as Infinite", The Review of Metaphysics, vol. 51, pp. 849-874.

Bassler, O. (1998b), “The Leibnizian Continuum in 1671”, en Studia Leibnitiana, 30:1, pp. $1-23$.

Bassler, O. (1999), “Towards Paris: The Growth of Leibniz's Paris Mathematics out of the Pre-Paris Metaphysics”, en Studia Leibnitiana 31:2, pp. 160-180.

Bassler, O. (2002), "Motion and Mind in the Balance: The Transformation of Leibniz's Early Philosophy”, en Studia Leibnitiana 34:2, pp. 221-231.

Beeley, P. (1996), Kontinuität und Mechanismus. Zur Philosohie des jungen Leibniz in ihrem ideengeschichtlichen Kontext, Stuttgart, Franz Steiner Verlag.

Bernstein, H. (1981), "Passivity and Inertia in Leibniz's Dynamics”, en Studia Leibnitiana 13:1, pp. 97-113.

Duchesneau, F. (1994), La dynamique de Leibniz, Paris, Vrin.

Fazio, R. (2016a), "La crítica de Leibniz a los números infinitos y su repercusión en la metafísica de los cuerpos", en Theoria: An International Journal for Theory, History and Foundations of Science, 31/2, pp. 159-175.

Fazio, R. (2016b), "Leibniz y la reforma de la metafísica: un análisis de la definición de sustancia como fuerza primitiva activa", en Revista Latinoamericana de Filosofia, Vol. XLII, N², pp. 149-170.

Fazio, R. (2017), "Movimiento, cuerpo y sustancia corpórea en Leibniz: la defensa de la relatividad del movimiento y su impacto en el desarrollo de la metafísica de los cuerpos", Eidos, N²6, pp. 238-267.

Garber, D. (1982), "Motion and Metaphysics in the Young Leibniz", en Leibniz: Critical and Interpretative Essays, Michael Hooker (ed.), Minneapolis, Univeristy of Minesota Press VI, pp. 160-184.

Garber, D. (1985), "Leibniz and the Foundations of Physics: The Middle Years," in The Natural Philosophy of Leibniz, edited by K. Okruhlik and J. R. Brown (ed), The Natural Philosophy of Leibniz, Dordrecht, Reidel, pp. 27-130.

Garber, D. (2004), “Leibniz and Fardella: Body, Substance, and Idealism,” P. Lodge (ed.), Leibniz and his Correspondents, Cambridge, Cambridge University Press, pp. 123-140.

Garber, D. (2009), Leibniz: Body, Substance, Monad, Oxford, Oxford University Press.

Hartz, G. (2007), Leibniz's Final System: Monads, Matter and Animals, London-New York, Routledge.

Hartz, G. (2008), “Why Corporeal Substances keep popping up in Leibniz's Later Philosophy”, British Journal for the History of Philosophy 6:2, 193-207.

Mercer, Ch. (2001), Leibniz's Methapysics: its origin and development, Cambridge, Cambridge University Press.

Moll, K. (1978; 1982; 1996), Der junge Leibniz, 3 Bd.; Stuttgart, Bad Cannstatt. 
Phemister, P. (2001), "Corporeal Substance and the Discourse on Metaphysics", Studia Leibnitiana, 33:1, pp. 68-85.

Phemister, P. (2005), Leibniz and the Natural World: Activity, Passivity and the Corporeal Substance, Dordrecht, Springer.

Robinet, A. (1986), Architectonique disjonctive, automates systémiques et idéalité transcendentale dans l' euvre de G. W. Leibniz, Paris, Vrin.

Rutherford, D. (2008), "Leibniz as Idealist", Oxford Studies in Early Modern Philosophy, vol. 4, pp. 141-190.

Smith, J. (2003), "Confused Perception and Corporeal Substance in Leibniz", The Leivniz Review, $\mathrm{n}^{\circ} 13$, pp. 45-64. 